\title{
Determinants of Short-Period Heart Rate Variability in the General Population
}

\author{
B. Kuch ${ }^{a, c}$ H.W. Hense ${ }^{a}$ R. Sinnreich ${ }^{d}$ J.D. Kark ${ }^{d}$ A. von Eckardstein ${ }^{b}$ \\ D. Sapoznikov ${ }^{d}$ H.-D. Bolte ${ }^{c}$
}

a Institute of Epidemiology and Social Medicine and bInstitute of Clinical Chemistry and Laboratory Medicine, University of Münster, and 'Department of Medicine I, Central Hospital Augsburg, Academic Training Clinic University Munich, Germany; ${ }^{\mathrm{D} D e p a r t m e n t}$ of Social Medicine and Department of Cardiology, Hadassah Medical Organization and Hebrew University-Hadassah School of Public Health, Jerusalem, Israel

\section{Key Words}

Autonomic nervous system · Heart rate variability · Population, general · Gender

\begin{abstract}
Decreased heart rate variability (HRV) is associated with a worse prognosis in a variety of diseases and disorders. We evaluated the determinants of short-period HRV in a random sample of 149 middle-aged men and 137 women from the general population. Spectral analysis was used to compute low-frequency (LF), high-frequency (HF) and total-frequency power. HRV showed a strong inverse association with age and heart rate in both sexes with a more pronounced effect of heart rate on HRV in women. Age and heart rate-adjusted LF was significantly higher in men and HF higher in women. Significant negative correlations of BMI, triglycerides, insulin and positive correlations of HDL cholesterol with LF and total power occurred only in men. In multivariate analyses, heart rate and age persisted as prominent independent predictors of HRV. In addition, BMI was strongly negatively associated with LF in men but not in women. We conclude that the more pronounced vagal influence in cardiac regulation in middle-aged women and the gender-different influence of heart rate and metabolic factors on HRV may help to explain the lower susceptibility of women for cardiac arrhythmias.
\end{abstract}

Copyright $\odot 2001$ S. Karger AG, Basel

\begin{tabular}{ll}
\hline KARGER & ( ) 2001 S. Karger AG, Basel \\
0008-6312/01/0953-0131\$17.50/0 \\
$\begin{array}{l}\text { Fax +4161306 12 34 } \\
\text { www.karger.com }\end{array}$ & $\begin{array}{l}\text { Accessible online at: } \\
\text { www.karger.com/journals/crd }\end{array}$
\end{tabular}

\section{Introduction}

Heart rate variability (HRV) has been recognized as a noninvasive tool for the assessment of cardiovascular autonomic balance. Recent investigations have shown that decreased HRV is associated with a worse prognosis in a variety of diseases and disorders $[1,2]$. Results from population-based follow-up studies revealed that lower HRV is associated with the risk of coronary heart disease and death $[3,4]$.

HRV can be assessed by time-domain analysis or by frequency-domain analysis. The latter enables us to calculate different components of the power spectrum by spectral density analysis. Thus, the high-frequency (HF) component, which measures the amplitude of sinus node respiratory arrhythmias, mainly depends upon the effects of vagal modulation on sinus node, whereas low-frequency power (LF) comprises both sympathetic and vagal oscillations; however, some authors suggest that LF represents predominantly sympathetic modulation [5]. Most research to date has been performed in cardiac patients or in high-risk individuals with diabetes or neurological disorders. There are only few studies that investigated the determinants and correlates of HRV as it occurs in the general population [6-8]. We present here results from a study that was carried out in middle-aged men and women from a representative population sample in southern Germany. 


\section{Subjects and Methods}

\section{Study Population}

The subjects of this analysis were participants of the German sample of a trinational population-based study [9]. Age-stratified random samples of men and women aged 45-65 years were drawn from the population of Augsburg (Germany). One hundred and fiftythree men and 143 women were examined after an overnight fast (participation rate $71 \%$ ).

\section{Interview and Physical Examination}

Interview questionnaires were employed to assess smoking habits, alcohol consumption, use of medications and the medical history. Body weight was measured in light clothing with precision to the nearest $0.1 \mathrm{~kg}$. Body height was measured without shoes to the nearest $0.5 \mathrm{~cm}$. Body mass index (BMI) was calculated as weight (in $\mathrm{kg}$ ) divided by height (in $\mathrm{m}$ ) squared. Obesity was defined according to National Institutes of Health Consensus Development Panel criteria as a BMI greater than $27.3 \mathrm{~kg} / \mathrm{m}^{2}$ in men and $27.8 \mathrm{~kg} / \mathrm{m}^{2}$ in women [10]. Blood pressure was measured 3 times on the right arm of sitting subjects by an oscillometric automated sphygmomanometer (BOSO Oscillomat) after a resting period of at least $5 \mathrm{~min}$. The average of the second and third measurement was used for this analysis. Hypertension was defined as systolic blood pressure $>140 \mathrm{~mm} \mathrm{Hg}$ or diastolic blood pressure $>90 \mathrm{~mm} \mathrm{Hg}$ or use of antihypertensive medication.

Fasting blood samples were drawn at the end of the physical examination. Serum and EDTA and citrated plasma samples were subsequently stored in liquid nitrogen $\left(-196^{\circ} \mathrm{C}\right)$ in Augsburg. The frozen samples were later transported on dry ice to the Institute of Arteriosclerosis Research Laboratory at the University of Münster, Germany. Concentrations of total cholesterol, HDL cholesterol, triglycerides and glucose were quantified using a Hitachi 917 autoanalyzer. Determinations of cholesterol, triglycerides and HDL cholesterol were controlled by the NHLBI Lipid Standardization Program organized by the Centers of Disease Control (CDC; Atlanta, Ga., USA). Insulin was measured by the use of a commercially available radioimmunoassay. Diabetes was defined as use of insulin or oral antidiabetic drugs or a fasting glucose $>126 \mathrm{mg} / \mathrm{dl}$ [11].

\section{Analysis of $H R V$}

Holter recording was carried out using a Marquette 8500 recorder. The recordings were undertaken in a quiet room under standardized conditions after a resting period of at least $10 \mathrm{~min}$. After connection of chest leads in the supine position the recordings were performed during $6 \mathrm{~min}$ of silent supine free breathing, and then $6 \mathrm{~min}$ of supine metronomic controlled breathing at 15 breaths per minute. Holter cassette tapes were analyzed on a Marquette series 8000 analysis system using two channels: a modified V5 lead and a modified V1 lead. Files with RR intervals and their annotations (normal beats, ectopic beats and artifacts) were transferred to a 486 personal computer through an RS-232 serial port using the XMODEM protocol. RR intervals were calculated utilizing a computer program and editing routines developed by Sapoznikov et al. [12]. Subjects with atrial fibrillation, excessive ectopic beats or technically inadequate recordings were excluded ( $n=4$ men and $n=6$ women). Five-minute epochs of heart rate as a function of time were used for power spectrum (power) analysis. Power analysis was performed with a 16th order autoregressive model and by solving the Yule-Walker equations with the Levinson algorithm [13]. Three frequency bands were computed: 0.0033 to $<0.04 \mathrm{~Hz}$ (very low), 0.04 to $<0.15 \mathrm{~Hz}$ (low), and 0.15 to $0.40 \mathrm{~Hz}$ (high). Although the very low frequency band is computed and it has been shown to be reliably measured in 5-min recordings [8], its use in short-term recordings $(<5 \mathrm{~min})$ generally has not been recommended [5]. In the present paper we, therefore, report the area of the LF band in milliseconds squared, the area of the HF band in milliseconds squared and the total power between 0.0033 and $0.40 \mathrm{~Hz}$ (total power) in milliseconds squared. To express the relative contribution of the respective frequency bands to total power, normalized units were calculated as the ratio between the power of each component and the total power less the very low component multiplied by $100[14,15]$. Two time domain measures, the standard deviation (SD) of RR intervals and the root mean square of successive differences in RR intervals (RMSSD), were also calculated. Because of their high correlation with the spectral parameters $(r=$ 0.99 for SD with total power and $r=0.95$ of RMSSD with HF) they did not provide additional information and, therefore, are not considered in the present paper. Finally, to standardize respiratory rate because differences in breathing frequency could influence differences in heart rate dynamics, metronomic controlled breathing was performed [8].

\section{Statistical Analysis}

Results are given as mean values. All variables were normally distributed except measures of HRV as well as concentrations of insulin and triglycerides. Accordingly, not normally distributed variables were naturally log-transformed. Univariate relations were assessed by Pearson's product-moment correlation. Groups were compared with Student's two-tailed unpaired t test for continuous variables and by $\chi^{2}$ tests for prevalences. On the basis of the assumption of detecting a difference of at least $0.3 \mathrm{~ms}^{2}$ in log-transformed HRV parameters between men and women at a $80 \%$ power, it was estimated that a sample size of more than 200 is sufficient. At the same power, for subgroup comparisons, e.g. diabetics versus nondiabetics in men and women, respectively, the sample sizes of men and women were sufficient enough for detecting a difference of $0.4 \mathrm{~ms}^{2}$ (about $10 \%$ of the respective means). Linear regression modeling was used for multivariate analysis. In order to assess gender differences and to account for the potential confounding effect of other HRV determinants, we tested the gender difference by use of an interaction term in a linear regression model encompassing the data of men and women while controlling for all factors univariately associated with one of the HRV indices. Forward stepwise multiple regression analysis was used to select variables independently related to the HRV parameters. To avoid failing to take account of potential HRV determinants due to low numbers, a significance level of 0.1 was applied for the univariate analyses of table 2 ; otherwise $p$ values $<0.05$ were considered as statistically significant. All analyses were carried out with the $\mathrm{SAS}^{\circledR}$ System for Windows Release 6.11.

\section{Results}

Table 1 shows the characteristics of our study population. Men had a less favorable cardiovascular risk profile than women with higher levels of blood pressure, glucose, insulin, triglycerides and higher mean HDL cholesterol. Men also smoked more often. 
Table 1. Characteristics of the study population

\begin{tabular}{lccl}
\hline & $\begin{array}{l}\text { Men } \\
(\mathrm{n}=149)\end{array}$ & $\begin{array}{l}\text { Women } \\
(\mathrm{n}=137)\end{array}$ & $\begin{array}{l}\text { p value for } \\
\text { difference }\end{array}$ \\
\hline Age, years & $56.0 \pm 5.9$ & $55.9 \pm 5.6$ & 0.9 \\
Height, cm & $172.7 \pm 6.6$ & $161.1 \pm 6.3$ & 0.0001 \\
BMI, kg/m ${ }^{2}$ & $27.9 \pm 4.0$ & $27.4 \pm 4.9$ & 0.28 \\
Systolic BP, mm Hg & $131.1 \pm 17.0$ & $127.1 \pm 16.8$ & 0.06 \\
Diastolic BP, mm Hg & $82.0 \pm 9.2$ & $76.8 \pm 9.1$ & 0.0001 \\
Heart rate, beats/min & $68.8 \pm 10.1$ & $70.9 \pm 10.1$ & 0.09 \\
Total cholesterol, mg/dl & $238 \pm 47$ & $235 \pm 42$ & 0.5 \\
HDL-cholesterol, mg/dl & $48.5 \pm 14.0$ & $59.8 \pm 14.5$ & 0.0001 \\
Glucose, mg/dl & $111 \pm 24$ & $104 \pm 26$ & $<0.02$ \\
Insulin, IU & $119.1 \pm 61.9^{\mathrm{a}}$ & $100.9 \pm 60.2^{\mathrm{a}}$ & $<0.01$ \\
Triglycerides, mg/dl & $120.3 \pm 70.4^{\mathrm{a}}$ & $87.9 \pm 66.5^{\mathrm{a}}$ & 0.0001 \\
\hline Hypertension, \% & $43(64)$ & $33(45)$ & 0.10 \\
Obesity, \% & $51(76)$ & $45(62)$ & 0.33 \\
Smoking, \% & $24(36)$ & $10(13)$ & 0.002 \\
Diabetes, \% & $10(15)$ & $4(6)$ & 0.11 \\
\hline
\end{tabular}

Values in the upper part represent mean $\pm \mathrm{SD}$. Values in parentheses represent number. a Geometric mean with approximation of standard deviation.

Table 2. Pearson correlation coefficients between HRV parameters and the other variables

\begin{tabular}{lllllllll}
\hline & \multicolumn{2}{l}{ Men $(\mathrm{n}=149)$} & & \multicolumn{2}{l}{ Women $(\mathrm{n}=137)$} \\
\cline { 2 - 5 } \cline { 6 - 8 } & LF power & HF power & total power & & LF power & HF power & total power \\
\hline Heart rate & $-0.29^{*}$ & $-0.35^{* *}$ & $-0.37^{* *}$ & & $-0.42^{* *}$ & $-0.64^{* *}$ & $-0.50^{* *}$ \\
BMI & $-0.26^{* *}$ & $-0.17^{*}$ & $-0.28^{* *}$ & & 0.01 & -0.07 & -0.01 \\
Systolic BP & -0.07 & -0.03 & -0.09 & & -0.08 & -0.08 & -0.08 \\
Diastolic BP & -0.04 & -0.01 & -0.06 & & -0.10 & $-0.16^{*}$ & $-0.15^{\circ}$ \\
Age & $-0.16^{* *}$ & $-0.18^{*}$ & $-0.19^{*}$ & & $-0.16^{\circ}$ & $-0.15^{\circ}$ & $-0.15^{\circ}$ \\
Height & $0.16^{*}$ & $0.19^{*}$ & $0.15^{*}$ & & 0.04 & 0.12 & 0.08 \\
Total cholesterol & 0.04 & 0.08 & 0.02 & & -0.09 & -0.09 & -0.10 \\
HDL cholesterol & $0.17^{*}$ & $0.15^{*}$ & $0.16^{*}$ & & -0.04 & 0.01 & -0.01 \\
Triglycerides & $-0.17^{*}$ & -0.05 & $-0.17^{*}$ & & 0.12 & 0.00 & 0.01 \\
Glucose & -0.12 & -0.12 & $-0.13^{\circ}$ & & 0.08 & 0.06 & 0.06 \\
Insulin & $-0.20^{*}$ & -0.04 & $-0.21^{*}$ & & 0.01 & -0.02 & 0.00 \\
\hline
\end{tabular}

${ }^{\circ} \mathrm{p}<0.1 ; * \mathrm{p}<0.05 ; * * \mathrm{p}<0.001$

LF power $=$ Area at low frequency $(0.04$ to $<0.15 \mathrm{~Hz})$; HF power $=$ area at high frequency $(0.15$ to $<0.40)$; total power $=$ total power spectrum $(0.0033$ to $<0.40 \mathrm{~Hz})$. The values for the HRV parameters, insulin and triglycerides were naturally log-transformed.

\section{Short-Term HRV Measured under Free Breathing \\ Conditions}

Correlation coefficients between HRV indices obtained with free breathing and cardiovascular risk factors and anthropometric variables were assessed in men and women (table 2). Heart rate was inversely associated with LF, HF and total power $(\mathrm{p}<0.001)$ and these correlations were particularly pronounced in women (correlation coefficients from 0.42 to 0.60 ). The inverse associations with age were less marked ( $p<0.05$ in men, $p<0.1$ in women). 
Table 3. Crude and adjusted mean values $( \pm$ SD) of LF, HF and total power in men and women

\begin{tabular}{|c|c|c|c|c|c|c|}
\hline & \multicolumn{3}{|l|}{ Crude } & \multicolumn{3}{|c|}{ Adjusted for age and heart rate } \\
\hline & $\operatorname{men}(n=149)$ & women $(n=137)$ & $\mathrm{p}$ value & men $(n=149)$ & women $(n=137)$ & $\mathrm{p}$ value \\
\hline LF power, $\mathrm{ms}^{2}$ & $5.04 \pm 0.94$ & $4.72 \pm 1.07$ & $<0.01$ & $5.00 \pm 0.92$ & $4.76 \pm 0.92$ & $<0.03$ \\
\hline $\mathrm{LF}$ as NU, $\%$ & $67.6 \pm 14.7$ & $58.5 \pm 16.6$ & $<0.0001$ & $67.9 \pm 14.7$ & $58.1 \pm 16.5$ & $<0.0001$ \\
\hline HF power, $\mathrm{ms}^{2}$ & $4.22 \pm 1.05$ & $4.33 \pm 1.08$ & 0.38 & $4.17 \pm 0.90$ & $4.39 \pm 0.90$ & $<0.05$ \\
\hline $\mathrm{HF}$ as NU, $\%$ & $32.4 \pm 14.7$ & $41.5 \pm 19.6$ & $<0.0001$ & $32.1 \pm 14.6$ & $41.8 \pm 19.6$ & $<0.0001$ \\
\hline Total power, $\mathrm{ms}^{2}$ & $6.19 \pm 0.83$ & $6.03 \pm 0.89$ & 0.1 & $6.16 \pm 0.75$ & $6.07 \pm 0.76$ & 0.31 \\
\hline
\end{tabular}

LF power = Area at low frequency $(0.04$ to $<0.15 \mathrm{~Hz})$; HF power = area at high frequency $(0.15$ to $<0.40)$; total power $=$ total power spectrum $(0.0033$ to $<0.40 \mathrm{~Hz})$; the values for the areas and total power are naturally log-transformed and expressed in $1 \mathrm{n} \mathrm{ms}^{2}$; $\mathrm{NU}=$ normalized units, calculated as the ratio between the power of each component and the total power less the very low component multiplied by 100 .

Table 4. Age- and heart rate-adjusted mean values ( \pm SD) of LF, HF and total power in relation to obesity and smoking

\begin{tabular}{|c|c|c|c|c|c|c|}
\hline & \multicolumn{3}{|c|}{$\operatorname{Men}(n=149)$} & \multicolumn{3}{|c|}{ Women $(n=137)$} \\
\hline & no & yes & $\mathrm{p}$ value & no & yes & $\mathrm{p}$ value \\
\hline Obese & $\mathrm{n}=75$ & $\mathrm{n}=74$ & & $\mathrm{n}=73$ & $\mathrm{n}=64$ & \\
\hline LF power, $\mathrm{ms}^{2}$ & $5.24 \pm 0.93$ & $4.80 \pm 0.90$ & $<0.01$ & $4.76 \pm 1.21$ & $4.67 \pm 0.87$ & 0.56 \\
\hline HF power, $\mathrm{ms}^{2}$ & $4.37 \pm 0.98$ & $4.18 \pm 1.08$ & $<0.07$ & $4.42 \pm 1.15$ & $4.23 \pm 0.99$ & 0.21 \\
\hline Total power, $\mathrm{ms}^{2}$ & $6.36 \pm 0.79$ & $6.02 \pm 0.82$ & $<0.01$ & $6.10 \pm 0.99$ & $6.05 \pm 0.76$ & 0.26 \\
\hline Smoking & $\mathrm{n}=113$ & $\mathrm{n}=36$ & & $\mathrm{n}=124$ & $\mathrm{n}=13$ & \\
\hline LF power, $\mathrm{ms}^{2}$ & $5.10 \pm 0.97$ & $4.86 \pm 0.98$ & 0.19 & $4.73 \pm 1.09$ & $4.65 \pm 0.76$ & 0.77 \\
\hline HF power, $\mathrm{ms}^{2}$ & $4.24 \pm 0.98$ & $4.19 \pm 1.23$ & 0.82 & $4.39 \pm 1.07$ & $3.81 \pm 0.90$ & $<0.02$ \\
\hline Total power, $\mathrm{ms}^{2}$ & $6.23 \pm 0.80$ & $6.09 \pm 0.92$ & 0.38 & $6.05 \pm 0.91$ & $5.87 \pm 0.58$ & 0.45 \\
\hline
\end{tabular}

LF power $=$ Area at low frequency $(0.04$ to $<0.15 \mathrm{~Hz})$; HF power $=$ area at high frequency $(0.15$ to $<0.40)$; total power $=$ total power spectrum $(0.0033$ to $<0.40 \mathrm{~Hz})$; the values for the areas and total power are naturally logtransformed and expressed in $\ln \mathrm{ms}^{2}$.

In men, the BMI was significantly related to a decreased $\mathrm{HF}, \mathrm{LF}$ and total power $(\mathrm{p}<0.01)$. Furthermore, triglycerides and insulin as well as low HDL cholesterol levels were all inversely related to LF and total power in men $(\mathrm{p}<0.05)$. In women, only diastolic blood pressure could be identified as a very modest correlate of HRV.

Though having similar total power, men and women differed markedly in the components of HRV (table 3 ). Mean LF was significantly higher in men $(p<0.01)$. This difference persisted after adjustment for age and heart rate, whereas unadjusted HF power was not significantly different between men and women; however, adjusting for age and heart rate revealed higher mean HF in women $(p<0.05)$. Expression in relative terms revealed that the proportionate contributions of HF and LF to total power were highly different in men and women $(\mathrm{p}<0.001)$.

We further assessed HRV in subjects by a risk factor group. Obesity was a pronounced correlate of generally reduced HRV in men only (table 4). Women smoking more than 10 cigarettes a day showed an impaired HF power $(\mathrm{p}<0.02)$. While diabetes was associated with lower crude HF power in men and women (4.29 \pm 1.01 vs. $3.82 \pm 1.17 \mathrm{~ms}^{2}$ in diabetic vs. nondiabetic men, respectively, $\mathrm{p}=0.05 ; 4.39 \pm 1.03$ vs. $3.64 \pm 1.54 \mathrm{~ms}^{2}$ for women, respectively, $\mathrm{p}<0.04)$ control for age and heart rate rendered these differences insignificant $(4.25 \pm 1.00$ vs. 
Table 5. Regression coefficients $(\beta)$ and partial $\mathrm{R}^{2}$ values of factors related to $L F$ and HF power in the final model after a forward stepwise procedure

\begin{tabular}{|c|c|c|c|c|c|c|}
\hline & \multicolumn{3}{|c|}{$\operatorname{Men}(n=149)$} & \multicolumn{3}{|c|}{ Women $(n=137)$} \\
\hline & $\beta$ & partial $\mathrm{R}^{2}$ & $\mathrm{p}$ value & $\beta$ & partial $\mathrm{R}^{2}$ & $\mathrm{p}$ value \\
\hline \multicolumn{7}{|l|}{ LF power, $\mathrm{ms}^{2}$} \\
\hline Heart rate & -0.025 & 0.08 & 0.0003 & -0.045 & 0.19 & 0.0001 \\
\hline BMI & -0.05 & 0.053 & 0.003 & - & - & - \\
\hline Age & -0.026 & 0.026 & 0.036 & -0.032 & 0.023 & 0.048 \\
\hline \multicolumn{7}{|l|}{ HF power, $\mathrm{ms}^{2}$} \\
\hline Heart rate & -0.03 & 0.125 & 0.0001 & -0.07 & 0.42 & 0.0001 \\
\hline Age & -0.03 & 0.038 & 0.01 & -0.029 & 0.02 & 0.017 \\
\hline HDL & 0.01 & 0.019 & 0.07 & - & - & - \\
\hline
\end{tabular}

LF power $=$ Area at low frequency $(0.04$ to $<0.15 \mathrm{~Hz}) ; \mathrm{HF}$ power $=$ area at high frequency $(0.15$ to $<0.40)$; the values for the areas are naturally log-transformed and expressed in $1 \mathrm{~ns}^{2}$.

Table 6. Regression coefficients $(\beta)$ and partial $\mathrm{R}^{2}$ values of factors related to $\mathrm{LF}$ and HF power in the same model as in table 5 with HRV parameters measured under metronomic breathing conditions

\begin{tabular}{|c|c|c|c|c|c|c|}
\hline & \multicolumn{3}{|c|}{$\operatorname{Men}\left(n=146^{a}\right)$} & \multicolumn{3}{|c|}{ Women $(\mathrm{n}=137)$} \\
\hline & $\beta$ & partial $\mathrm{R}^{2}$ & $\mathrm{p}$ value & $\beta$ & partial $\mathrm{R}^{2}$ & $\mathrm{p}$ value \\
\hline \multicolumn{7}{|l|}{ LF power, $\mathrm{ms}^{2}$} \\
\hline Heart rate & -0.015 & 0.023 & 0.05 & -0.04 & 0.16 & 0.0001 \\
\hline BMI & -0.072 & 0.11 & 0.0001 & - & - & - \\
\hline Age & -0.027 & 0.027 & 0.036 & - & - & - \\
\hline \multicolumn{7}{|l|}{ HF power, $\mathrm{ms}^{2}$} \\
\hline Heart rate & -0.038 & 0.086 & 0.0001 & -0.075 & 0.36 & 0.0001 \\
\hline BMI & -0.10 & 0.128 & 0.0001 & -0.027 & 0.014 & 0.075 \\
\hline Insulin & -0.0018 & 0.031 & 0.017 & - & - & - \\
\hline HDL & - & - & - & 0.017 & 0.033 & 0.008 \\
\hline
\end{tabular}

LF power $=$ Area at low frequency $(0.04$ to $<0.15 \mathrm{~Hz})$; HF power $=$ area at high frequency $(0.15$ to $<0.40)$; the values for the areas are naturally log-transformed and expressed in $\ln \mathrm{ms}^{2}$.

a Number is reduced to 146 because 3 men were not able to follow metronomic breathing.

$4.10 \pm 1.19 \mathrm{~ms}^{2}$ in diabetic vs. nondiabetic men, respectively, $\mathrm{p}=0.5 ; 4.37 \pm 1.02$ vs. $3.93 \pm 1.61 \mathrm{~ms}^{2}$ for women, respectively, $\mathrm{p}=0.11$; data not shown). Similarly, HRV was not markedly different in men or women with and without hypertension (data not shown). Finally, HRV of women older than 50 years on hormonal replacement therapy (HRT; $\mathrm{n}=95)$ did not differ significantly from that in women not taking HRT $(\mathrm{n}=22): 4.71 \pm 1.16$ vs. $4.39 \pm 0.84 \mathrm{~ms}^{2}(\mathrm{p}=0.23)$ for $\mathrm{LF} ; 4.30 \pm 1.09$ vs. $3.91 \pm$ $0.84 \mathrm{~ms}^{2}(\mathrm{p}=0.12)$ for $\mathrm{HF}$, and $6.00 \pm 0.96$ vs. $5.76 \pm$
$0.63 \mathrm{~ms}^{2}(\mathrm{p}=0.26)$ for total frequency, respectively (data not shown).

In the final model of the multivariate analysis, heart rate, age and - for men only - BMI remained as predictors of LF power and heart rate, age and - of borderline significance in men only - HDL cholesterol for HF power. Heart rate alone accounted for $19 \%$ of the variance of LF power and $42 \%$ of the variance of HF power in women. Its impact was much less prominent in men (table 5). 
The modification of the effects of heart rate or BMI on HRV by sex was assessed specifically by modeling statistical interaction terms. We found that the association of heart rate with decreased LF and HF power in women was significantly stronger than that in men $\left(\mathrm{R}^{2}=0.21, \mathrm{p}<0.05\right.$ for the interaction term of $\mathrm{LF}$ and $\mathrm{R}^{2}=0.32, \mathrm{p}<0.01$ for $\mathrm{HF}$, respectively). Conversely, the association of BMI with LF in men was significantly stronger than that in women $\left(\mathrm{R}^{2}=0.21, \mathrm{p}<0.05\right.$ for the interaction term $)$.

\section{Short-Term HRV under Metronomic Breathing Conditions}

Under metronomic breathing conditions results were mostly similar to those obtained with unrestricted breathing. The association between BMI and decreased HRV in men emerged as even slightly stronger (table 6). Hypertensive men revealed significantly lower $\operatorname{HF}(p<0.02$, adjusted for age and heart rate). Likewise, diabetic women showed decreased HF power $(\mathrm{p}<0.02$, adjusted for age and heart rate).

\section{Discussion}

In this study, we aimed to identify determinants of HRV in men and women from a representative sample of the general population. We show that middle-aged men have higher average LF power as compared to HF components which prevail in women: these differences became particularly obvious when heart rate was accounted for in the analyses or when indices were expressed in proportional terms. Our findings extend previous reports of male/female differences in HRV [7, 8, 14, 16-18] by delineating that risk factors affect cardiac autonomic regulation in a sex-specific way.

\section{Heart Rate and HRV}

Consistent with numerous previous reports, we found a strong relation between heart rate and HRV indices [8, $15,19]$. We report here that the influence of heart rate on HRV seems significantly more pronounced in women than men. Generally, the inverse influence of heart rate on HF was remarkably higher than that on LF power. This finding is consistent with the observation that raised heart rates commonly indicate sympathetic activation [20] and might, therefore, be expected to decrease the LF component to a lesser extent. Moreover, it has been shown that a high resting heart rate may also result from a decreased vagal activity [21] confirming our observation of an inverse association of heart rate with HF power.

\section{Anthropometry, Cardiovascular Risk Factors and $H R V$}

Age was inversely associated with all HRV indices and the strength of this association was similar in men and in women. In contrast to a previous study [19], but consistent with another one [8], age contributed much less to the variance of HRV than heart rate. This discrepancy may be explained by the restricted age range in our study sample.

BMI plays a central role in the metabolic syndrome (i.e. effects of metabolic factors such as lipids, glucose and insulin) which is believed to increase the susceptibility to severe arrhythmias and sudden death by increasing sympathetic activity [22]. The influence of adiposity on autonomic cardiac function has rarely been investigated. The few studies to date have been inconsistent with findings of reduced RR interval variation in obese men [23] while other studies in subjects younger than 40 years found no [7] or negative associations with HRV and BMI in both genders [24]. In accordance with our study, previous studies observed stronger inverse associations of HRV or heart rate with BMI in men than in women $[15,20]$.

In univariate analyses, the biochemical correlates of the metabolic syndrome, HDL cholesterol, triglycerides and insulin as well as diabetes, were associated with decreased HRV. It has been shown that with the clustering of metabolic syndrome disorders HRV indices decrease [2]; however, no sex-specific analyses were reported. In the present study we show that after including BMI in the multivariate model these factors made only a small independent contribution to the overall variance of HRV in the general population.

Similarly, smoking which in our study as well as in another population [7] showed an inverse association with HF power in women, was eliminated in multivariate analyses. It also appears that hypertension as a major cardiovascular risk factor plays no relevant role as a determinant of HRV.

\section{Mechanisms of HRV Differences between Men and Women}

It has also been suggested previously that estrogens have a cardioprotective effect in young women [25] as well as in older, postmenopausal women [15]. Huikuri et al. [14] reported a higher parasympathetic activity in postmenopausal women given hormonal replacement therapy. We were unable to confirm an influence of hormone replacement therapy in our study. However, the generally increased parasympathetic activity in women, as reflected by a higher average HF component, may partly explain

\footnotetext{
$\overline{136} \quad \overline{\text { Cardiology 2001;95:131-138 }}$
}

Kuch/Hense/Sinnreich/Kark/ von Eckardstein/Sapoznikov/Bolte 
the relative protection of women from cardiovascular disease $[8,26]$.

\section{Study Limitations}

There are limitations to the present study. HRV was measured during the morning hours, over a short period of $5 \mathrm{~min}$, in resting conditions and in the supine position. Thus, it may not be representative of the sympathetic/ parasympathetic balance over the 24-hour period. However, standardized application of procedures for crosscenter comparison was the predominant concern in the original study protocol, an objective which is not achievable with 24-hour recording. Though the reliability of HRV measurement was not assessed in the present study, a previous investigation in an Israeli sample using the same protocol reported relatively high reliability coefficients for short-time HRV recordings [8]. Therefore, we believe that the associations between established risk factor levels and resting HRV can be validly assessed with our shortterm measurements. In addition, residual confounding and unmeasured variables may account for some of the sex differences in HRV. For example, physical fitness may affect HRV because physical activity is associated with an accentuation of vagal activity [27]. On the other hand, men in the Augsburg population tended to be physically more active than women [28] which the HRV gender differences may even have underestimated. Finally, the power of the study may not have been sufficient enough to detect smaller differences $\left(<0.4 \mathrm{~ms}^{2}\right)$ between risk factor groups in men and women, or in HRT use among older women, respectively.

In conclusion, our findings add to the increasing evidence that men show a higher sympathetic and women an enhanced vagal control of heart rate. Factors related to the metabolic syndrome determine HRV in men; however, the overall association of HRV with cardiovascular risk factors was rather weak. Heart rate is of outstanding importance for both LF and HF components, more so in women than men. Whether the gender differences in the regulation of autonomic cardiac function are related to the lower susceptibility of women to arrhythmias and cardiac death requires further study.

\section{Acknowledgments}

We thank Dr. H. Roth and Prof. Dr. J. Gostomzyk from the Gesundheitsamt of the city of Augsburg for their logistical help.

This study was supported by a grant from the German-Israeli Foundation for Scientific Research and Development, grant No. I-253-135.02/92.

\section{References}

1 Kleiger RE, Miller JP, Bigger JT, Moss AJ, the Multicenter Post-Infarction Research Group: Decreased heart rate variability and its association with increased mortality after acute myocardial infarction. Am J Cardiol 1987;59:256262.

2 Liao D, Sloan RP, Cascio W, Folsom A, Liese AD, Evans GW, Cai J, Sharrett AR: Multiple metabolic syndrome is associated with lower heart rate variability. The Atherosclerosis Risk in Communities Study. Diabetes Care 1998; 21:2116-2122.

3 Tsuji H, Larson G, Venditti FJ, Manders ES, Evans JC, Feldman CL, Levy D: Impact of reduced heart rate variability on risk for cardiac events - The Framingham Study. Circulation 1996;94:2850-2855.

4 Liao D, Cai J, Rosamund WD, Barnes RW, Hutchinson RG, Whitsel EA, Rautaharju P, Heiss G: Cardiac autonomic function and incident coronary heart disease: A populationbased case-cohort study. The ARIC Study. Am J Epidemiol 1997;145:696-706.
5 Task Force of the European Society of Cardiology and the North American Society of Pacing and Electrophysiology: Heart rate variability. Standards of measurement, physiological interpretation, and clinical use. Circulation 1996; 93:1043-1065.

6 Liao D, Barnes RW, Chambless LE, Simpson RJ, Sorlie P, Heiss G: Age, race and sex differences in autonomic cardiac function measured by spectral analysis - The ARIC study. Am J Cardiol 1995;76:906-912.

7 Kupari M Virolainen J, Koskinen P, Tikkanen MJ: Short-term heart rate variability and factors modifying the risk of coronary artery disease in a population sample. Am $\mathrm{J}$ Cardiol 1993; 72:897-903.

8 Sinnreich R, Kark JD, Friedländer Y, Sapoznikov D, Luria MH: Five minute recordings of heart rate variability for population studies: Repeatability and age-sex characteristics. Heart 1998;80:156-162.
9 Bobak M, Hense HW, Kark J, Kuch B, Vojtisek P, Sinnreich R, Gostomzyk J, Bui M, von Eckardstein A, Junker R, Fobker M, Schulte H, Assmann G, Marmot M: An ecological study of determinants of coronary heart disease rates: $\mathrm{A}$ comparison of Czech, Bavarian and Israeli men. Int J Epidemiol 1999;28:437-444.

10 National Institutes of Health Consensus Development Conference Statement: Health Implications of Obesity. Ann Int Med 1985;103: 1073-1077.

11 American Diabetes Association: Report of the expert committees on the diagnosis and classification of diabetes mellitus. Diabetes Care 1997;20:1183-1197.

12 Sapoznikov D, Luria MH, Mahler Y, Gotsman MS: Computer processing of artifact and arrhythmias in heart rate variability analysis. Comput Methods Programs Biomed 1992;39: 75-84.

13 Marple SL: Digital Spectral Analysis. Englewood Cliffs, Prentice Hall, 1987.

14 Huikuri HV, Pikkujämsä M, Airaksinen J, Ikäheimo MJ, Rantala AO, Kauma H, Lilja M, Kesäniemi A: Sex-related differences in autonomic modulation of heart rate in middle-aged subjects. Circulation 1996;94:122-125. 
15 Ramaekers D, Ector H, Aubert AE, Rubens A, Van de Werf F: Heart rate variability and heart rate in healthy volunteers -- Is the female autonomic system cardioprotective? Eur Heart J 1998;19:1334-1341.

16 Ryan SM, Goldberger AL, Pincus SM, Mietus J, Lipsitz LA: Gender- and age-related differences in heart rate dynamics: Are women more complex than men? J Am Coll Cardiol 1994; 24:1700-1707.

17 Molgaard J, Hermansen K, Bjerregaard P Spectral components of short-term RR interval variability in healthy subjects and effects of risk factors. Eur Heart J 1994; 15:1174-1183.

18 Yamasaki Y, Kodama M, Matsuhisa M, Kishimoto M, Ozaki H, Tani A: Diurnal heart rate variability in healthy subjects: Effects of aging and sex differences. Am J Physiol 1996; 271(suppl 40): H303-H310.
19 Tsuji H, Venditti FJ, Manders ES, Evans JC, Larson MG, Feldman CL, Levy D: Determinants of heart rate variability. J Am Coll Cardiol 1996;28:1539-1546.

20 Palatini P: Heart rate as a cardiovascular risk factor. Eur Heart J 1999; 1(suppl B):B3-B9.

21 Hayano J, Yamada A, Mukai S, Sakakibara Y, Yamada M, Ohte N, Hashimoto T, Fujinami T, Takata K: Severity of coronary atherosclerosis correlates with the respiratory component of heart rate variability. Am Heart J 1991;121: 1070-1079.

22 Reaven GM, Lithell H, Landsberg L: Hypertension and associated metabolic abnormalities - The role of insulin resistance and the sympathoadrenal system. N Engl J Med 1996; 334:374-381.

23 Peterson HR, Rotschild M, Weinberg CR, Fell RD, McLeish KR, Pfeifer MA: Body fat and the activity of the autonomic nervous system. N Engl J Med 1988;318:1077-1083.
24 Jensen-Urstad M, Jensen-Urstad K, Ericson $\mathrm{M}$, Johansson $\mathrm{J}$ : Heart rate variability is related to leucocyte count in men and to lipoproteins in women in a healthy population of 35-yearold subjects. J Intern Med 1998;243:33-40.

25 Clarkson TB: Pathophysiologic processes of atherogenesis; in Eaker ED, Packard B, Wenger NK, Clarkson TB, Tyroler HA (eds): Coronary Heart Disease in Women. New York, Haymarket Doyma, 1986, pp 11-19.

26 Kannel WB, Schatzkin A: Sudden death: Lessons from subsets in population studies. J Am Coll Cardiol 1994;24:1700-1707.

27 Aubert AE, Ramaekers D, Cuche Y, Lysens R, Ector H, Van de Werf F: Effect of long term physical training on heart rate variability. Comput Cardiol 1996;27:17-20.

28 Stender M, Hense HW, Döring A, Keil U: Physical activity at work and cardiovascular disease risk: Results from the MONICA Augsburg Study. Int J Epidemiol 1993;22:664-670. 